\title{
Theranostics
}

Review

\section{Application of Molecular Ultrasound for Imaging Integrin Expression}

\author{
Fabian Kiessling ${ }^{\bowtie}$, Jessica Gaetjens and Moritz Palmowski \\ Department of Experimental Molecular Imaging, RWTH Aachen University, Germany
}

Corresponding author: Prof. Dr. Fabian Kiessling, Department of Experimental Molecular Imaging, RWTH-Aachen University, Pauwelsstrasse 20, 52074 Aachen, Germany, Tel: 0049 (0) 2418036124 ; fax: +49 (0) 241 8082442; e-mail: fkiessling@ukaachen.de

(C) Ivyspring International Publisher. This is an open-access article distributed under the terms of the Creative Commons License (http://creativecommons.org/ licenses/by-nc-nd/3.0/). Reproduction is permitted for personal, noncommercial use, provided that the article is in whole, unmodified, and properly cited.

Published: 2011.02 .01

\begin{abstract}
Stabilized microbubbles with a size between I-5 $\mu \mathrm{m}$ are used as ultrasound contrast agents in the clinical routine. They have shown convincing results for the vascular characterization of tissues as well as in echocardiography. Due to their size, microbubbles strictly remain intravascular where they can be detected with high sensitivity and specificity. This qualifies them for intravascular molecular imaging. Many studies have been published reporting on the successful use of microbubbles conjugated to specific ligands for target identification in vivo. Among them, there are several promising examples on how to use molecular ultrasound for the imaging of integrin expression. This review provides an overview on the composition of ultrasound contrast agents that can be used for molecular imaging and their detection by ultrasound using destructive and non destructive methods. Furthermore, concrete examples are given on the use of molecular ultrasound to characterize integrin expression on vessels. These cover oncological applications where integrin targeted microbubbles were used to identify and characterize tumor angiogenesis and to assess tumor response to antiangiogenic drugs as well as to radiotherapy. In addition, increased accumulation of integrin targeted microbubbles was found during vascular reformation in ischemic tissues as well as in vulnerable atherosclerotic plaques. In summary, there is clear evidence from preclinical studies that integrin targeted ultrasound imaging is a valuable tool for the characterization of a broad spectrum of diseases. Thus, more efforts should be put into translating this promising technology into the clinics.
\end{abstract}

Key words: Molecular ultrasound; Microbubble; Integrin; Angiogenesis; Therapy response.

\section{The role of ultrasound in tumor imaging}

In the clinical routine ultrasound often is the first imaging modality applied to patients. Reasons are its low costs, the high mobility and the lack of exposure of the patient to radioactive radiation. Furthermore, ultrasound is a real time imaging modality, which further makes this imaging tool suitable for interventional procedures (e.g. biopsy).

Besides providing anatomical information ultrasound is capable of displaying the perfused vas- culature and allows deriving functional vascular parameters - even without the need of injecting contrast material. Non contrast enhanced vascular imaging is based on the Doppler principle, which is also important for targeted ultrasound. In ultrasound the Doppler Effect describes the frequency shift of an acoustic wave by its reflection from a moving element [1]. If the object is moving towards to the transducer, the frequency increases and if it moves away, it decreases. In blood vessels acoustic pulse waves are mainly scattered by blood cells, which form the major 
blood cell fraction. The frequency shifts can be color coded and overlaid with the morphologic ultrasound images to visualize vessels and their flow velocities. Unfortunately, micro vessels with very slow flow velocities of blood cells can not be detected. Here ultrasound contrast agents can be used and these have proven to be valuable tools to detect angiogenic tumors and to characterize liver lesions by its vascularization [2].

Nevertheless, a local increase in vascularization can have many reasons reaching from cancer to autoimmunological and infectious disorders. Furthermore, the clearance time of ultrasound contrast agents from the blood is fast and thus labeling of these lesions persists only for a short period of time. This complicates the use of contrast agents during interventions. Thus, the availability of molecular ultrasound contrast agents providing longer local contrast and giving more information about the molecular background of the lesion are of high clinical interest. In this context, integrins have proven to be excellent markers of angiogenic vessels and their downregulation during anti-angiogenic therapies seems to indicate therapy response reliably. Therefore, ultrasound contrast agents targeting integrins can be considered to be exquisite tools to characterize angiogenic lesions, to demarcate these lesions during image guided interventions and to track pharmacological tumor therapy response $[3,4]$.

There are several reasons why these targeted ultrasound contrast agents are not in the clinics yet: First, the development costs for diagnostic agents are high and the more specific a contrast agent is, the smaller is the market. Therefore, pharmaceutical companies are cautious to develop such products for the clinics.

Second, ultrasound is user dependent and thus reproducibility of measurements is limited. Fortunately, novel automated 3D acquisition techniques have been introduced in the clinics by several companies recently, which certainly will have positive impact on the establishment of targeted ultrasound.

Third, there is some competition with other molecular imaging modalities (such as positron emission tomography). Personally, we do not consider this conflict to be relevant since ultrasound fills niches in real time imaging, bedside imaging and interventional imaging, while most other imaging modalities have their strengths in whole body imaging.

Additionally, there are concerns about the safety of ultrasound contrast agents. Indeed, there are reports on immunological reactions and even on lethal complications after the administration of ultrasound contrast agents in patients with severe cardiac disor- ders. However, a causal connection between these deaths and the administration of ultrasound contrast agents has not been proven, yet. Furthermore, immunological reactions appear much less often after injection of ultrasound contrast agents than after injection of X-ray or MRI contrast agents. Therefore, although ultrasound contrast agents can be considered to be relatively save, one has to keep in mind that all administered drugs can have side effects and that the indication for their application should always be made thoughtfully.

\section{Microbubbles as contrast agents}

On ultrasound images the contrast depends on absorption, reflection and scattering of the ultrasound pulse by tissue elements. An ultrasound contrast agent thus has to be a stronger acoustic reflector than the environment or even act as an acoustic emitter. It may also change the spectrum of the ultrasound pulse (non linear reflection) and thus generate its own specific fingerprint.

In general, molecules that reflect the ultrasound pulse linearly can be nano- to micrometer scaled and acoustically will behave similar to erythrocytes. However, they can not be detected with high sensitivity and specificity.

In contrast, taking into account the frequencies in which clinical ultrasound operates $(2-20 \mathrm{MHz})$ a microbubble of 1-3 $\mu \mathrm{m}$ in diameter can be optimally excited (and / or even destroyed) by ultrasound pulses and thus will provide a much stronger signal. Currently all commercial ultrasound contrast agents consist of stabilized microbubbles [5].

Ultrasound contrast agents can be classified according to the rigidity of their shell. Soft-shell microbubbles have either no coating or are covered by a thin monolayer of surfactant molecules such as palmitic acid or phospholipids and are very sensitive to pressure changes. Hard-shell microbubbles have a more rigid shell made of polymers or denaturized albumin, which can increase their stability to a certain degree. Both microbubble classes can be filled with either air or heavy gases such as sulfohexafluoride or perfluorocarbon. The advantage of heavy gases is the longer lasting stability of the gas bubble in the blood and the longer blood half life of the ultrasound contrast agent in the body. This is particularly important for soft shell bubbles, which are more vulnerable than hard shell bubbles. Thus most of them contain heavy gases. An overview on the commercially developed microbubbles and its characteristics is given in Table 1, which was taken from a publication of Kiessling and colleagues [5]. 
Table I. Overview on ultrasound contrast agents, which were developed in respect of a clinical application [5].

\begin{tabular}{|c|c|c|c|}
\hline Name & Manufacture & Gas core & Shell composition \\
\hline Imagify $^{\mathrm{TM}}$ & Acusphere & Decafluorobutane & poly-L-lactide co-glycide \\
\hline Albunex ${ }^{\circledR}$ & Mallinckrodt & Air & Albumin \\
\hline BiSphere $^{\mathrm{TM}}$ & Point Biomedical & Air & Polymer/Bilayer \\
\hline $\mathrm{BR} 14^{\circledR}$ & Bracco & Perfluorocarbon & Phospholipid \\
\hline BY963 & Nycomed & Air & Lipid \\
\hline Cardiosphere & Point Biomedical & Nitrogen & Polylactid/Albumin \\
\hline Definity ${ }^{\circledR}$ & DuPont/BMS & Octafluoropropane & Liposome \\
\hline Echogen & Sonus & Dodecafluoropentane & Surfactant \\
\hline Echovist $^{\circledR}$ & Schering & Air & No shell (galactose matr \\
\hline Imagent $^{\circledR}$ & IMCOR & Perfluorohexane & Surfactant \\
\hline Levovist $^{\circledR}$ & Schering & Air & Palmitic acid \\
\hline MP1950 & Mallinckrodt & Perfluorobutane & Phospholipid \\
\hline Myomap & Quadrant & Air & Albumin \\
\hline Optison ${ }^{\circledR}$ & GE Healthcare & Octafluoropropane & Albumin \\
\hline PESDA & $\begin{array}{l}\text { Porter (Univ. of } \\
\text { Nebraska) }\end{array}$ & Perfluorocarbon & Albumin \\
\hline Quantison $^{\mathrm{TM}}$ & Quadrant & A & A \\
\hline Sonavist & Schering & Air & Polycyanoacrylate \\
\hline Sonazoid $^{\mathrm{TM}}$ & GE Healthcare & Perfluorocarbon & Lipid \\
\hline Sonovue ${ }^{\circledR}$ & Bracco & Sulphur hexafluoride & Phospholipid \\
\hline
\end{tabular}

The design of targeted ultrasound contrast agents is not significantly different from these regular, non-specific perfusion agents, however, two major additional requirements have to be considered:

1. Attachment of specific ligands to the surface of the microbubble surface has to be possible.

2. The blood half life of the microbubble must be long enough to enable binding and accumulation at the target. Furthermore, the target persistence time has to be long enough to generate a suitable time window for its detection.

For the coupling of binding moieties amino- or carboxylic groups should be available at the microbubble surface. These can be used to couple streptavidin or biotin. Since biotin binds to streptavidin in a non covalent but very stable manner, biotinylated antibodies or peptides can then be linked to the microbubble (Fig. 1). This conjugation route is chosen by most groups working on targeted ultrasound. How- ever, from the clinical perspective this concept is not translatable since streptavidin is immunogenic in humans. As a consequence for the clinical application alternative conjugation routes have to be used. Direct coupling of ligands can be performed but often is complex from the chemical point of view. Poly-ethylene-glycol (PEG) can facilitate coupling, increase hydrophilicity and improve the target affinity of the molecular microbubble. Alternatively click chemistry can be used. This has the advantage of facilitating the use of different ligands without the need for time consuming individual optimization of coupling strategies.

A broad variety of different ligands can be coupled to microbubbles reaching from antibodies and their fragments to small peptides, peptidomimetics, aptamers and lectines. With respect to integrins targeting cyclic RGD peptides and av $\beta 3$-binding antibodies have been used so far (specified below). 


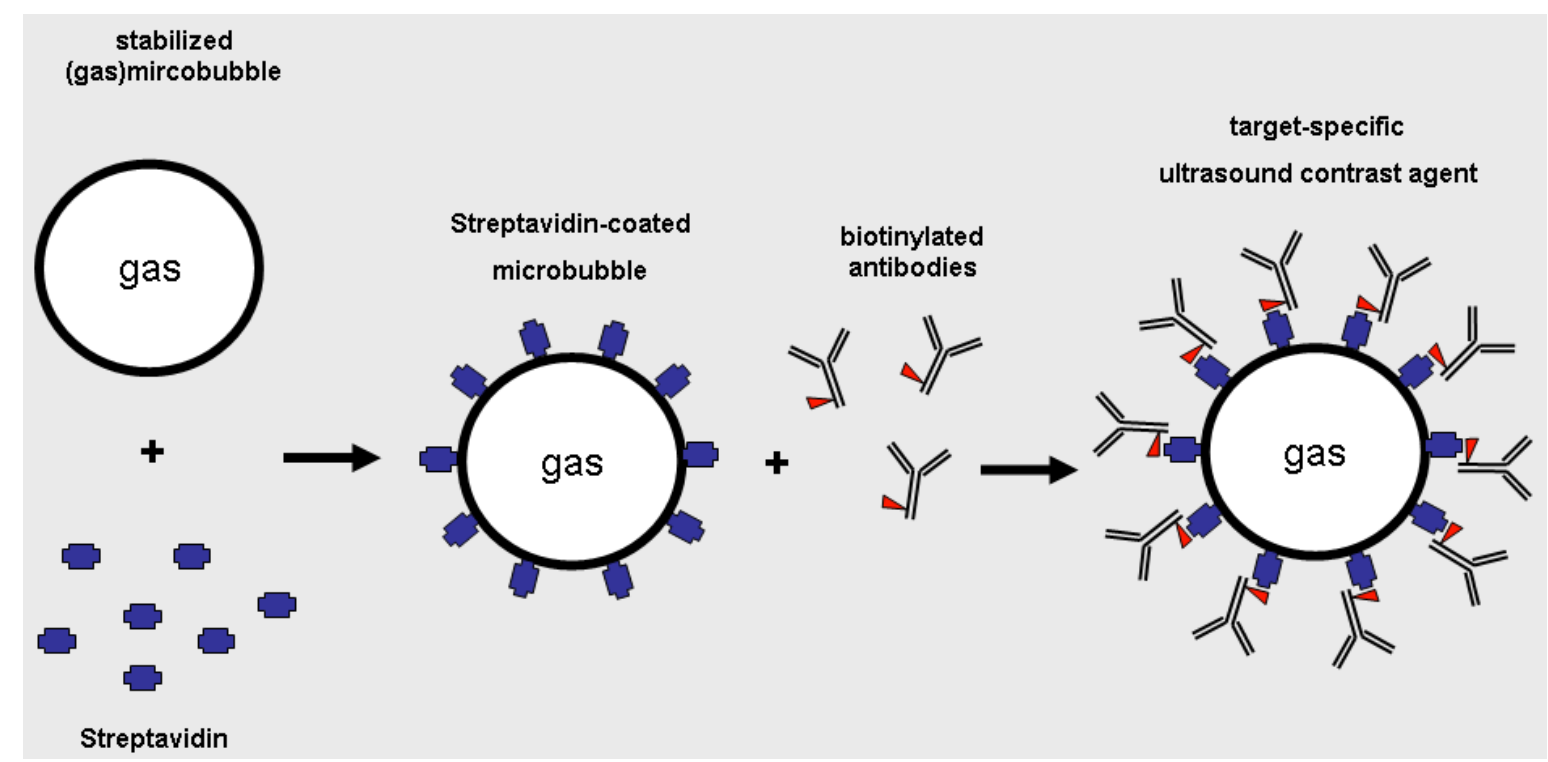

Figure I. Synthesis of target specific ultrasound contrast agents.

\section{Detection techniques}

Stationary microbubbles can be detected by different strategies: The simplest approach is to measure the relative contrast enhancement within a two-dimensional ultrasound image after the freely circulating microbubbles have been cleared from the blood (which usually takes few minutes) (Fig. 2).

Alternatively, the subharmonic and harmonic echoes can be used to detect microbubbles by their specific fingerprints. Even pulse inversion techniques can be applied, which effectively suppress the tissue background [5]. These approaches work most favorably at low mechanical indexes - meaning at low ultrasound pulse energy, which is sufficient to excite the microbubble without destroying it.

If high acoustic pressures are applied the microbubble disintegrates, which is termed "destructive imaging". During microbubble destruction, the air rapidly streams out of the collapsing microbubble shell, which generates a strong non linear signal. This signal can be detected by Doppler and is determined "Loss of Correlation". Since thereby every microbubble will only be counted once, exquisite options for the quantification of the microbubble concentration in the tissue are given. Unfortunately, the thickness of an ultrasound slice is depth dependent and certainly larger than the diameter of one microbubble. Also from the echo characteristics it is difficult to distinguish whether the signal within a voxel originates from only one or from several microbubbles. Saturation of the Doppler-signal is reached rapidly, which complicates quantification of stationary microbubble concentrations. Reinhardt and coworkers partially solved this problem by using a novel method determined as "Sensitive Particle Acoustic Quantification (SPAQ)" [6] (Fig. 3). SPAQ is a 3-dimensional scan procedure with overlapping image slice acquisitions. For this purpose the scanned object or the transducer are coupled to a servo-motor and moved in micrometer steps during image acquisition. An ultrasound energy pulse capable of destroying the microbubbles is applied. During the first destructive image microbubbles burst in a broad slice and quantification is difficult. However, after a micrometer scaled shift between the object and the transducer new $\mathrm{mi}$ crobubbles will only be destroyed in the non-overlapping distance. Thus, the next and also all subsequent ultrasound scans will only capture microbubbles from very thin excitation slices in which single microbubbles are detected. 


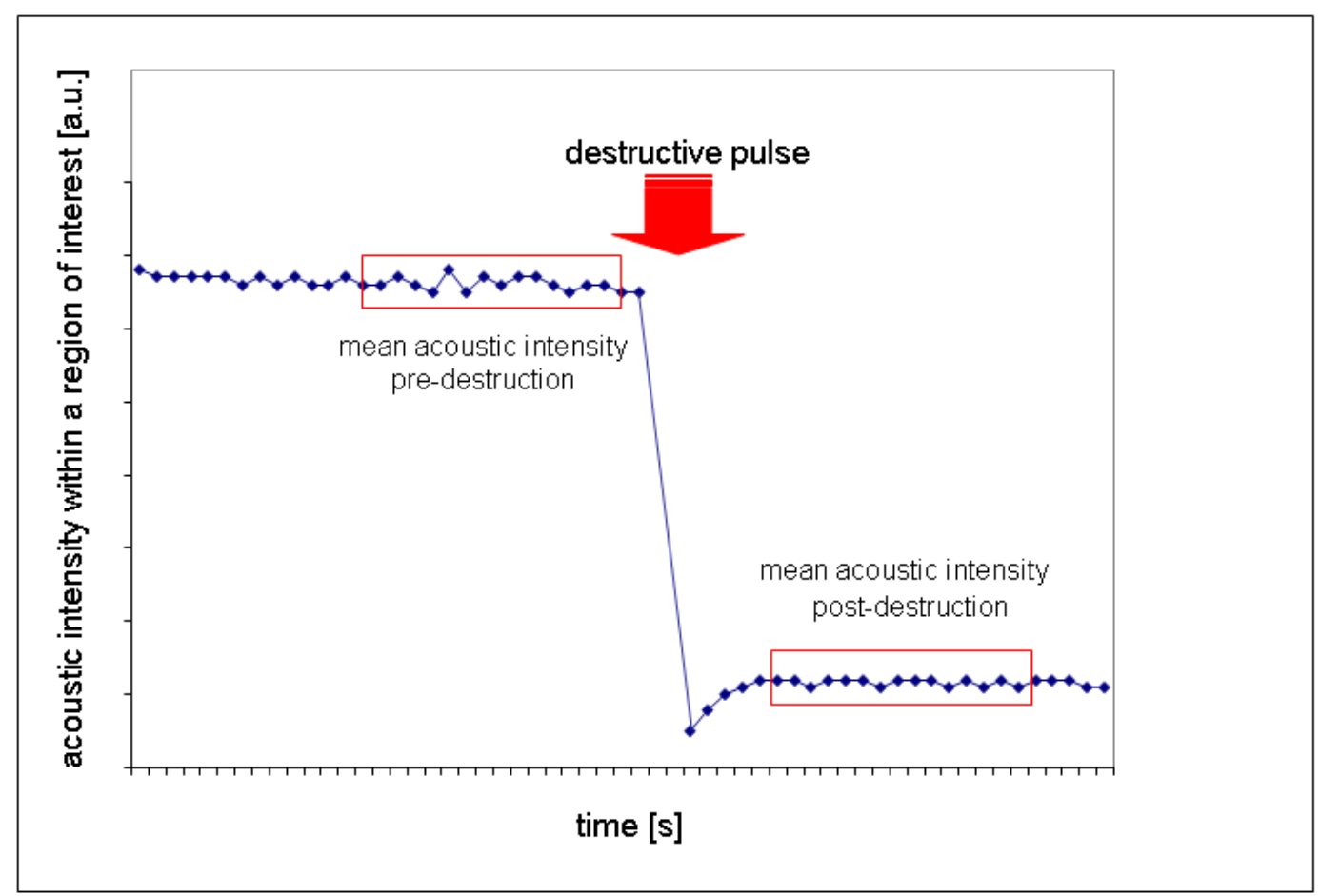

Figure 2. Non-destructive two-dimensional technique to assess the accumulation of targeted microbubbles.

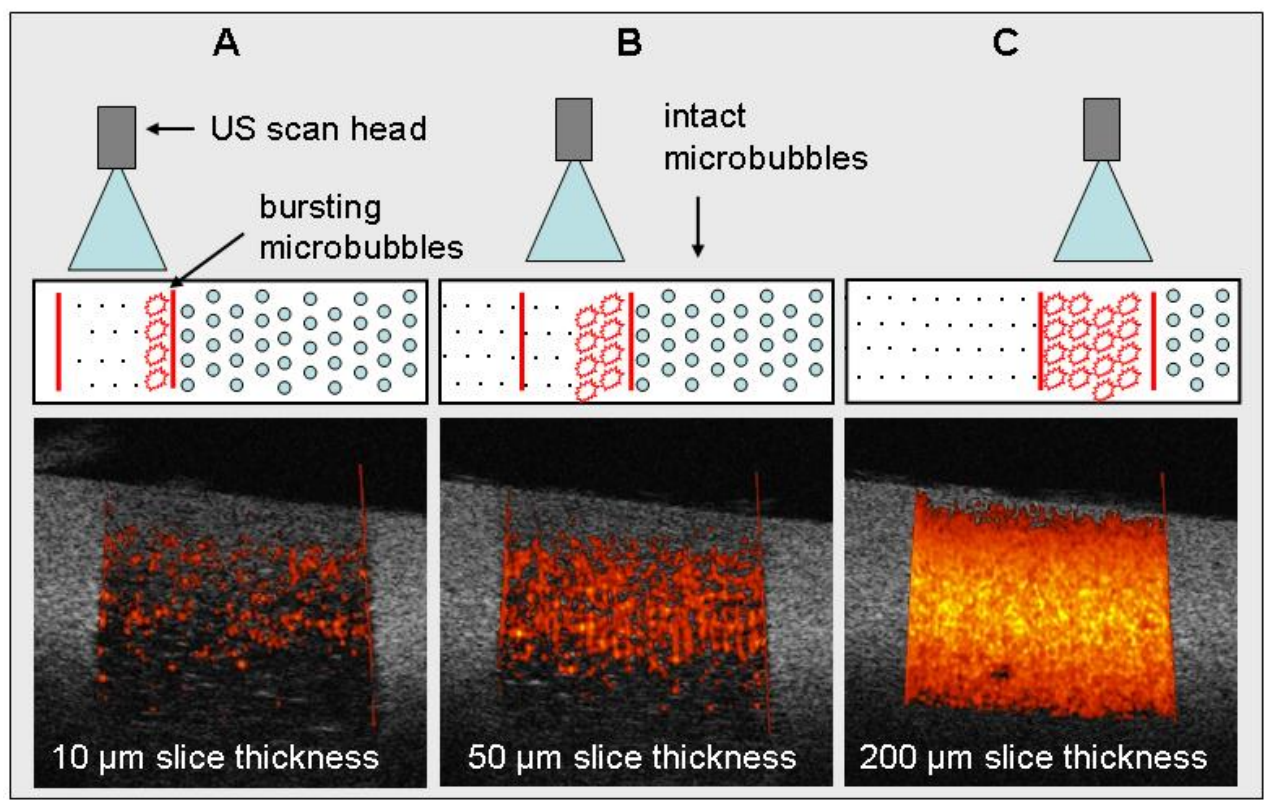

Figure 3. Three-dimensional quantification of stationary contrast agents using the sensitive particle acoustic quantification technology (SPAQ [6]). Drawings illustrate the principle of SPAQ. If an ultrasound transducer moves over an object (here: Agar-phantom containing microbubbles), the ultrasound beam destroys the microbubbles within the acoustic field. (A) Low frame-to-frame displacement between the single ultrasound pulses results in a high SPAQ resolution providing good discrimination of single signals. (B and C) Higher displacements (= larger SPAQ-slices) produce lower SPAQ resolutions finally resulting in an overlay of single SAE signals. "Proof-of-principle" in an agar phantom filled with microbubbles. Adapted from reference 16 with permission. 


\section{Applications}

av $\beta 3$ integrin has been intensively studied as a target for imaging inflammation or tumor angiogenesis. Like VEGFR2, av $\beta 3$ integrin is highly expressed on activated endothelium and almost absent on the remaining vessels [7]. Thus, it is an ideal target for the diagnosis of vessels activated by inflammation or tumor angiogenesis. However, when using ultra- sound contrast agents it is important to note that ultrasound contrast agents remain within the vessels, due to their size in the range of micrometers. Therefore, only targets expressed by endothelial cells can be addressed. Different binding ligands have been explored for targeting av $\beta 3$ integrin, as summarized in Table 2.

Table 2. Overview on studies about molecular ultrasound imaging of integrins

$\begin{array}{lcl}\begin{array}{l}\text { Author } \\ \text { Leong-Poi et al. [8] }\end{array} & \begin{array}{l}\text { Year } \\ 2003\end{array} & \begin{array}{l}\text { Ligands } \\ \text { Echistatin and } \\ \text { antibodies }\end{array} \\ \begin{array}{l}\text { Ellegala et al. [10] } \\ \text { Dayton et al. [11] }\end{array} & 2003 & \begin{array}{l}\text { Echistatin } \\ \text { cyclic RGD-peptides } \\ \text { and antibodies } \\ \text { Echistatin }\end{array} \\ \text { Leong-Poi et al. [9] } & 2004 & \text { Antibodies } \\ \text { Willmann et al. [12] } & 2008 & \text { Cyclic RGD-peptides } \\ \text { Palmowski et al. [14] } & 2008 & \text { Cyclic RGD-peptides } \\ \text { Palmowski et al. [15] } & 2009 & \end{array}$

Application

- Cremaster muscle of mice treated with FGF-2 and

- s.c. implanted FGF-2 enriched matrigel plugs in mice

- human gliomas orthotopically implanted in rats

- Cell monolayers in vitro

- Hindlimb ischemia in rats during endogeneous and therapeutic arteriogenesis

- Cell monolayers

- human ovarian cancer cells s.c. implanted in mice

- Human squamous cell carcinomas in mice during tumor progression and antiangiogenic treatment

- Rat prostate cancer s.c. implanted in rats during treatment with charged particles (heavy ions)

Leong-Poi and colleagues used monoclonal antibodies against murine av or echistatin, a viper venom disintegrin containing the well known RGD (arginine-glycine-aspartic acid) sequence [8]. Antibody-bearing and echistatin-conjugated microbubbles exhibited high potential to image activated endothelium in subcutaneously implanted Matrigel plugs enriched with fibroblast growth factor-2 (FGF-2). Microbubble retention inside the microvasculature was much higher as compared to non-targeted microbubbles, however, no significant differences in the retention of both types of targeted microbubbles were observed. Thus, monoclonal antibodies against murine av and echistatin can be used for targeted imaging approaches.

In another study, Leong-Poi and colleagues applied echistatin-conjugated microbubbles to assess endogeneous and therapeutic arteriogenesis in a hindlimb ischemia model in rats [9]. They observed an increase in retention of integrin-binding microbubbles prior to a measurable increase in blood flow. The binding of targeted microbubbles was even greater in a treatment group (FGF-2 therapy). These results demonstrate the high potential of molecular ultra- sound imaging to elucidate regulatory mechanisms, preceding the structural changes assessable by functional imaging techniques (e.g. increased blood flow due to new vessels).

Echistatin was further used as a binding ligand to image av $\beta 3$ expression in human glioma cells implanted intracerebrally in rats [10]. In this study, the authors were able to demonstrate significant retention of targeted microbubbles within tumors, and also reported on increasing accumulation after 14 days of tumor growth. They correlated the changing marker expression to changes in blood volume and were the first to state that imaging should be performed in combination with assessment of microbubble retention and relative blood volume. Assessing both types of information permits a conclusion to be drawn as to whether the changing retention of microbubbles is related to a general change in the endothelial surface (e.g. relative blood volume) or to a real up- (or down-) regulation of the markers by the endothelial cells.

Dayton and coworkers did not use Echistatin but cyclic analogs of the RGD ligand as well as integrin binding antibodies. Both ligands showed specific adhesion to av $\beta 3$-expressing cell monolayers in vitro 
[11]. The idea of using cyclic RGD-peptides and antibodies has then been taken up by Willmann and colleagues who produced dual-targeted microbubbles [12]. Their contrast agents were coated by an equal amount of RGD-peptides and antibodies. They showed superior binding properties both in vitro and in vivo as compared to microbubbles conjugated either with antibodies or RGD-peptides alone. The principle of an enhanced binding of microbubbles coated with different ligands has been described by Rychak et al before [13]. They assumed that fast (but unstable) binding peptides enable a firmer binding by antibodies. The combination of antibodies with fast binding peptides may thus open the door to contrast agents with superior specificity to selected targets (e.g. integrins).

Another group investigated the effects of an anti-angiogenic treatment on the expression of av $\beta 3$ integrins (and VEGFR2) [14]. VEGFR2 antibodies and cyclic RGD-peptides served as ligands. They used a three-dimensional imaging technique (SPAQ, [6]), enabling the sequential administration of different batches of specific microbubbles over short time intervals. Palmowski and colleagues hereby demonstrated an upregulation of VEGFR2 and of $\operatorname{av} \beta 3$ integrin during the growth of untreated tumors, and a downregulation of both markers after antiangiogenic therapy. In a subsequent study, the same group observed an upregulation of integrin-expression after irradiation with charged particles in prostate tumors [15] (Fig. 4). The study was motivated by the upcoming demand for individualized treatments with combination of radiotherapy and targeted drugs. Knowledge about the behavior of molecular targets after irradiation might enable to define the optimal time points for administration of individualized therapy regimens.

Molecular ultrasound imaging proved to be a reliable tool not only for diagnosis of integrin expression in suspect tissues but also for assessing changes in the expression of av $\beta 3$ integrin during disease progression or after different forms of treatment.

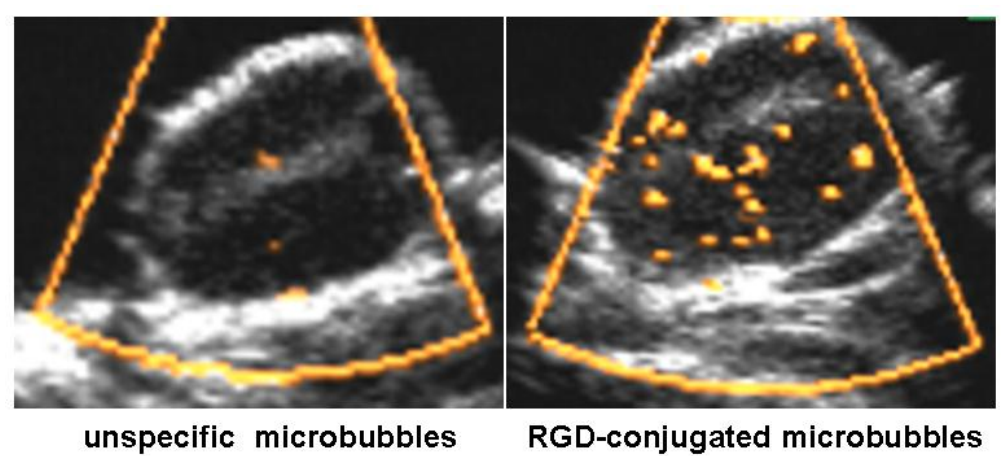

Figure 4. Subcutaneously implanted rat prostate carcinomas seven minutes after administration of unspecific microbubbles (left image) and RGD-coated microbubbles (right). Examinations were performed with the destructive imaging technique SPAQ [6]. After administration of specific ultrasound contrast agents, a large amout of Doppler signals can be detected within the tumor.

\section{Conflict of Interest}

The authors have declared that no conflict of interest exists.

\section{References}

1. Martinoli C, Pretolesi F, Crespi G, et al. Power Doppler sonography: clinical applications. Eur J Radiol 1998; 27 (Suppl 2): S133-40.

2. Lencioni R, Della Pina C, Crocetti L, Bozzi E, Cioni D. Clinical management of focal liver lesions: the key role of real-time contrast-enhanced US. Eur Radiol 2007; 17 (Suppl 6):F73-9.

3. Meloni MF, Livraghi T, Filice C, Lazzaroni S, Calliada F, Perretti L. Radiofrequency ablation of liver tumors: the role of microbubble ultrasound contrast agents. Ultrasound Q 2006; 22: 41-7.
4. Krix M. Quantification of enhancement in contrast ultrasound: a tool for monitoring of therapies in liver metastases. Eur Radiol 2005; 15 (Suppl 5): 104-8.

5. Kiessling F, Huppert J, Palmowski M. Functional and molecular ultrasound imaging: Concepts and contrast agents. Curr Med Chem 2009; 16: 627-42.

6. Reinhardt $\mathrm{M}$, Hauff $\mathrm{P}$, Briel A, et al. Sensitive particle acoustic quantification (SPAQ): a new ultrasound-based approach for the quantification of ultrasound contrast media in high concentrations. Invest Radiol, 2005; 40: 2-7.

7. Brooks PC, Clark RA, Cheresh DA. Requirement of vascular integrin $\alpha v \beta 3$ for angiogenesis. Science 1994; 264: 569-71.

8. Leong-Poi H, Christiansen J, Klibanov AL, Kaul S, Lindner JR. Noninvasive assessment of angiogenesis by ultrasound and microbubbles targeted to av-integrins. Circulation 2003; 107: 455-60.

9. Leong-Poi H, Christiansen J, Heppner P, et al. Assessment of endogenous and therapeutic arteriogenesis by contrast ultra- 
sound molecular imaging of integrin expression. Circulation 2005; 111: 3248-54.

10. Ellegala DB, Leong-Poi $\mathrm{H}$, Carpenter JE, et al. Imaging tumor angiogenesis with contrast ultrasound and microbubbles targeted to av $\beta 3$. Circulation 2003; 108: 336-41.

11. Dayton PA, Pearson D, Clark J, et al. Ultrasonic analysis of peptide- and antibody-targeted microbubble contrast agents for molecular imaging of av $\beta 3$-expressing cells. Mol Imaging 2004; 3: $125-34$.

12. Willmann JK, Lutz AM, Paulmurugan R, et al. Dual-targeted contrast agent for US assessment of tumor angiogenesis in vivo. Radiology 2008; 248: 936-44.

13. Rychak JJ, Li B, Acton ST, et al. Selectin ligands promote ultrasound contrast agent adhesion under shear flow. Mol Pharm 2006; 3: 516-24.

14. Palmowski M, Huppert J, Ladewig G, et al. Molecular profiling of angiogenesis with targeted ultrasound imaging: early assessment of antiangiogenic therapy effects. Mol Cancer Ther 2008; 7: 101-9.

15. Palmowski M, Peschke P, Huppert J, et al. Molecular Ultrasound Imaging of Early Vascular Response in Prostate Tumors irradiated with Carbon Ions. Neoplasia 2009; 11: 856-63.

16. Palmowski M, Lederle W, Bzyl J, Gaetjens J, Kiessling F. Molecular ultrasound imaging: principles and potential application in diagnosis and therapy monitoring of breast cancer. Eur Radiol 2009; 19 (Suppl 4): 862-7. 